\title{
SOLDER BUMPING - A FLEXIBLE JOINING APPROACH FOR THE PRECISION ASSEMBLY OF OPTOELECTRONICAL SYSTEMS
}

\author{
Erik Beckert ${ }^{\star}$, Thomas Burkhardt, Ramona Eberhardt, Andreas Tünner- \\ mann
}

Fraunhofer Institute for Applied Optics and Precision Engineering Dr. Erik Beckert, Fraunhofer IOF, Albert-Einstein-Strasse 7, 07745 Jena, Germany Phone: +493641807338, Fax: +493641807604

email: erik.beckert@iof.fraunhofer.de

\begin{abstract}
For microoptics and microsystem assembly, solder bumping is introduced as a flexible and high precise joining alternative to adhesive bonding, especially if adhesives limit the system performance in terms of long term, high temperature, vacuum or UV stability. The bumping technology, which is based on jetting the liquid solder onto wetting surfaces, can be parameter optimised to work in complex environments as well as with submicron accuracy.
\end{abstract}

Keywords optics, microassembly, laser beam soldering, bumping, jetting

\section{Motivation}

Joining of components is, next to an accurate alignment, the most challenging process in the precise microassembly of optical and optoelectronical systems, since it is the last step of the process chain to securely fixate the position of sensitive and functional relevant parts in the system and to connect them to respective thermal and electrical interfaces. Bonding with UV-curing adhesives has been the standard technology for this task, but is limited in terms of long term stability, radiation robustness and outgassing in vacuum environments. Force-based clamping technologies are not an appropriate alternative since miniaturised components and brittle materials often not allow for the adaption of clamping mounts.

Soldering, being a metal-based adhesion joining technology, can be such an alternative. With a wide variety of available, lead-free solder alloys that exhibit excellent and variable mechanical parameters solder joints provide creep-minimised, electrically and thermally conducting connections between a mounted part and its system environment. These joints are high temperature and cryogenic stable, but are non-transparent and thus cannot be used, in contrast to polymeric adhesives, within any optical beam propagation path. But of more importance is that soldering in mi-

Please use the following format when citing this chapter:

Beckert, E., Burkhardt, T,, Eberhardt, R., Tünnermann, A., 2008, in IFIP International Federation for Information Processing, Volume 260, Micro-Assembly Technologies and Applications, eds. Ratchev, S., Koelemeijer, S., (Boston: Springer), pp. 139-147. 
croassembly requires different reflow approaches than that known from electronics manufacturing. Due to sensitive parts and materials the reflow energy should be applied thermally and locally restricted, e.g. by laser. Second, fluxless processing is required to prevent any contamination of optical surfaces. Finally, solder application, its reflow and resolidification must not influence the alignment state of the component to be assembled outbound of tolerable limits that often are in the micron and submicron range. Solder bumping is a technology that provides all these features and incorporates the necessary equipment in one singular and flexible device.

\section{Solder Bumping - the process}

\section{2.l Technology basics}

Laser based Solder Bumping [1], shown schematically in Fig. 1, was developed to individually place bumps for the flip chip assembly of microelectronic circuits [2]. The patented process places bumps made of various soft solder alloys with melting temperatures ranging up to $280^{\circ} \mathrm{C}$ on different substrates usually without preheating the metallic wetting surface.

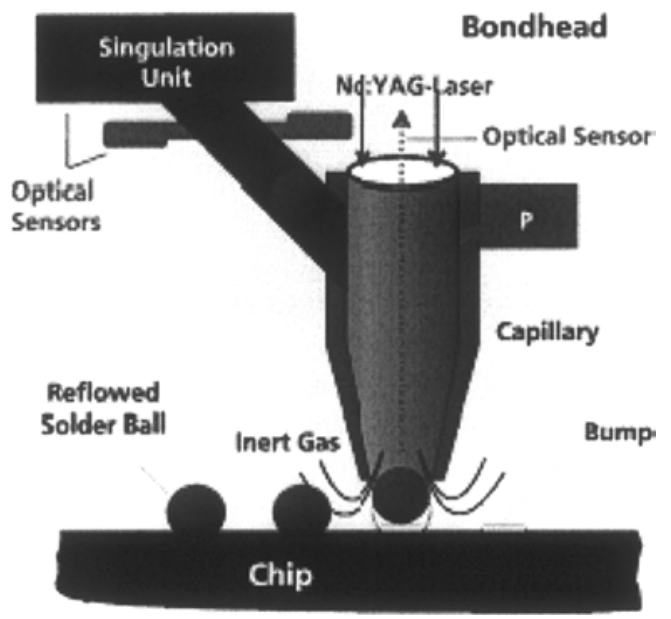

Fig. 1. Solder Bumping [1], Source: Pac Tech GmbH

In a reservoir the solder alloy is fed to the bumping device in the form of solid spheres with a uniform, narrowly $( \pm 3 \mu \mathrm{m})$ tolerated diameter, ranging from $100 \mu \mathrm{m}$ to $760 \mu \mathrm{m}$. A singulation unit transfers a sphere into the bumping capillary at the beginning of the bumping process. The inner diameter of the capillary is at its end smaller than the diameter of the sphere, so by gravity force the sphere gets locked at the outlet of the capillary. This locking state is further secured by applying nitrogen pressure from within the capillary, which now can be flexibly moved towards the desired bumping position, e.g. by a robot. It is noticeable that this positioning 
desired bumping position, e.g. by a robot. It is noticeable that this positioning movement, after locking the solder sphere at the outlet of the capillary, can incorporate all six degrees of freedom, especially tip and tilt (Fig. 2), enabling for the positioning of the capillary in even complex, miniaturised and three dimensional integrated system environments.

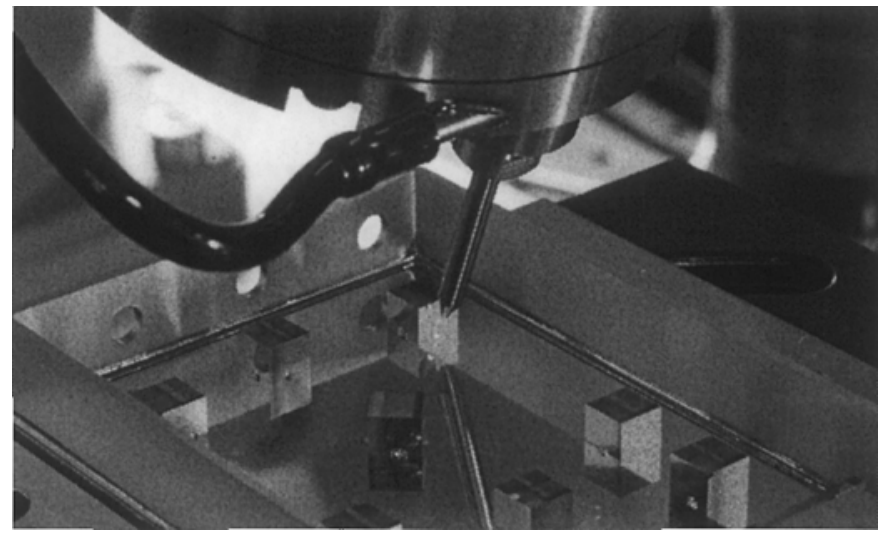

Fig. 2. Tilted capillary in a complex assembly environment

After the desired position is reached, a reflow laser is applied through the capillary. In the current setup a fibre coupled, pulsed Nd:YAG-laser with pulse widths ranging from $15 \mathrm{~ms}$ to $25 \mathrm{~ms}$ and pulse energies up to $4 \mathrm{~J}$ is used, but can be adapted to the actually chosen solder material and volume. The laser pulse liquidifies the solder volume and stores thermal energy within it. Now being liquid the solder gets pressed out of the capillary by the nitrogen pressure that also forms an inert atmosphere around it, and flies towards the substrate, while the laser beam during pulse time pre-heats the wetting area by exiting the now empty capillary outlet. $0.5 \mathrm{~mm}$ up to $6 \mathrm{~mm}$ free flow bumping distances for the solder spheres where demonstrated.

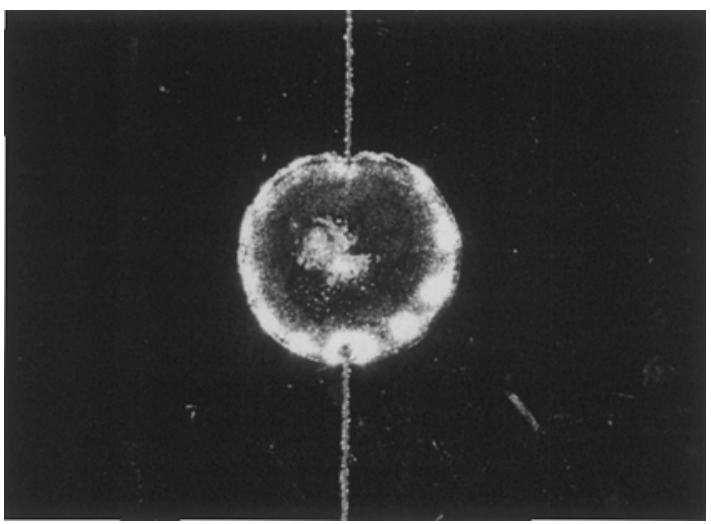

Fig. 3. Bump applied to vertically orientated wetting surfaces 
When the still liquid solder hits the wetting surface, it locally heats the area by giving its stored thermal energy away, thus realising a stable joint after resolidification by creating the necessary intermetallic phases. The wetting area does not necessarily have to be a horizontal or planar surface, but can be shaped in any geometry as long as the solder volume can reach the wetting surfaces on both parts to be joined. Bump application was demonstrated even on vertical surfaces (Fig. 3) and with joining gaps up to $100 \mu \mathrm{m}$ present.

\subsection{Parameter analysis}

Applying the bumping technology to the various circumstances during precision assembly of optoelectronic microsystems requires not only a fundamental understanding of the wetting physics of the liquid solder hitting a cold or only locally preheated metallic joining surface with different shape, but also an analysis of the various parameters that influence the bumping results. In terms of optics, the results are characterised by:

- dealigment during bumping (required usually to be in the micron and submicron range) due to solder resolidification and long term creep of the solder resulting from intermetallic phase changes and diffusion processes,

- mechanical stability of the joint including repeatability of the wetting behaviour under fluxless processing conditions,

- introduced mechanical stress and micro cracks, thus reduction of mechanical stability, due to thermal shock when liquid solder hits the wetting surface.

A robustness analysis according to Taguchi rules [3] of the bumping process under various conditions is currently carried out. In a more general investigation on the influencing factors a parameter field shown in Table 1 was generated. First experiments indicated, that bump distance and pulse energy, coupled with the deposited energy within the solder volume, are of the most sensitive parameters to be optimised in order to reach a minimised dealignment while securing wettability on different substrates without damaging the bulk material of the components to be joined [4]. 


\begin{tabular}{|c|c|c|}
\hline Parameter & Range & Influence \\
\hline \multicolumn{3}{|l|}{ Laser reflow parameters } \\
\hline Pulse energy & $0 \mathrm{~J}$ to $4 \mathrm{~J}$ & Thermal shock, wettability, \\
\hline Pulse width & $15 \mathrm{~ms}$ to $25 \mathrm{~ms}$ & Pre-heating of wetting surface \\
\hline \multicolumn{3}{|l|}{ Bump parameters } \\
\hline Solder sphere diameter & $100 \mu \mathrm{m}$ to $760 \mu \mathrm{m}$ & Pulse energy, dealignment \\
\hline Bump distance & $0.5 \mathrm{~mm}$ to $6 \mathrm{~mm}$ & Bump formation, Dealignment \\
\hline Bump direction & $0^{\circ}$ to approx $45^{\circ}$ & Bump formation \\
\hline Wetting area geometry & planar, complex & Bump formation \\
\hline Joining gap & up to approx. $100 \mu \mathrm{m}$ & Bump formation, Dealignment \\
\hline Nitrogen pressure & & $\begin{array}{l}\text { Bump formation, Wettability, } \\
\text { Delignment }\end{array}$ \\
\hline \multicolumn{3}{|c|}{ Material parameters solder and components to be joined } \\
\hline Solder melting temperature & $140^{\circ} \mathrm{C}$ to $280^{\circ} \mathrm{C}$ & Pulse energy \\
\hline Surface metallization & $\mathrm{Au}, \mathrm{AgPd}$, others & Wettability \\
\hline Substrate thermal conductivity & up to $400 \mathrm{~W} \mathrm{~m}^{-1} \mathrm{~K}^{-1}$ & Pulse energy, Wettability \\
\hline
\end{tabular}

Table 1. Parameter field for the bumping process

Fig. 4 demonstrates the result of an accuracy test with respect to the bumping distance. Motivation for this experiment was that in complex assembly environments it is often difficult to reach the desired joining area even with the small and up to $15 \mathrm{~mm}$ long bumping capillary. Thus it would be feasible to increase the bump distance as much as possible, so that only the flying solder volume after reflow would need a path through the environment. But investigations, where bumps were set into a target grid, led to the result that accuracy of setting the bump at the respective target decreases with increasing bumping distance. For a $400 \mu \mathrm{m}$ solder sphere a bump distance above approx. $5 \mathrm{~mm}$ becomes thus useless.
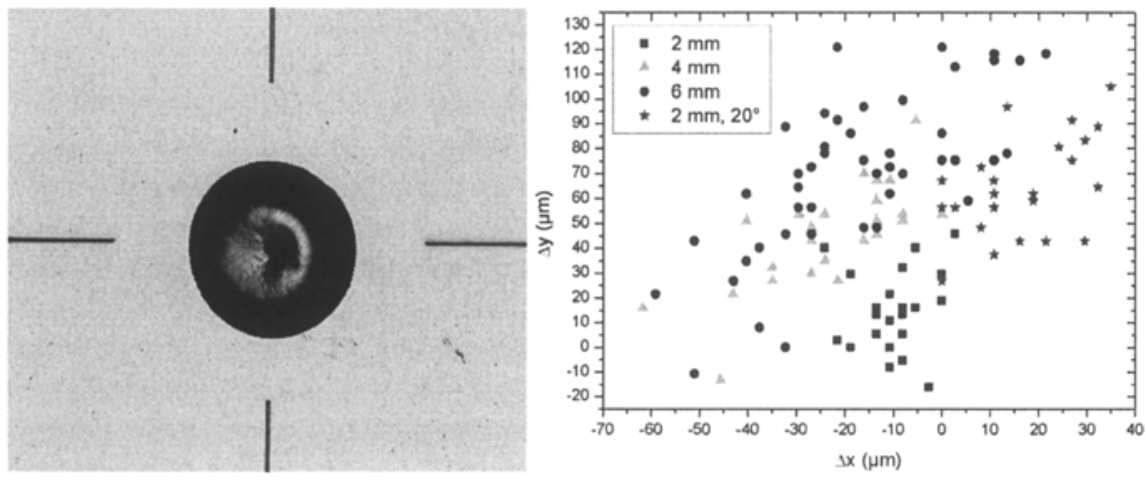

Fig. 4. Bump applied to a vertically orientated wetting target, accuracy with respect to different bumping distances 
Fig. 5 shows the link between pulse energy, wettability and damage to the components to be joined. Wettability becomes especially difficult if thermal conductivity of the bulk material is high, thus requiring a large amount of energy deposited in the solder volume to make wetting possible. In contrast, high pulse energy leads to an increased thermal shock when the liquid solder hits the wetting surface, which is not feasible for brittle materials like glass. Consequently, the optimisation goal is to find a pulse energy high enough to secure wettability and low enough to prevent damage to the components. This process window narrows if completely different materials have to be joined (e.g. a lens made of glass and a mount made of metal) and widens for smaller solder volume. In the current setup thus the solder sphere diameter was changed from initial $760 \mu \mathrm{m}$ to currently $400 \mu \mathrm{m}$. It was found that even on difficult wetting surfaces with underlying copper bulk material joints could be created with the solder fracture strength being the limiting factor for mechanical stability.
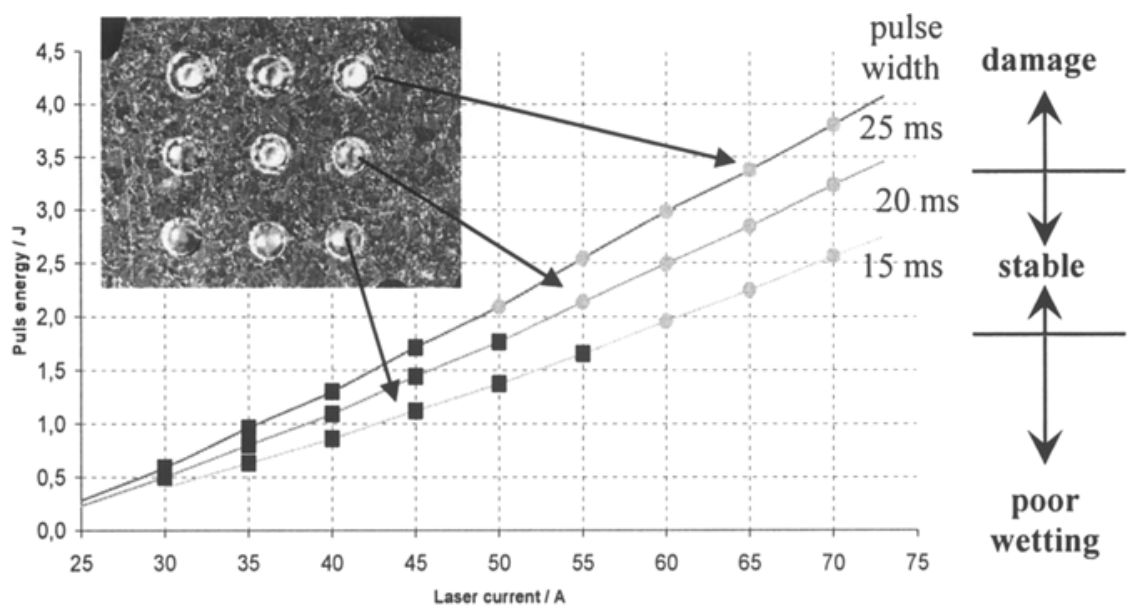

Fig. 5. Pulse energy process window for a copper substrate

\section{Application example}

A very common task in the precise assembly of optoelectronical systems is the accurate alignment and fixation of collimating lenses in front of laser source. Socalled aspherical Fast Axis Collimators (FAC) create a parallel beam from the divergent propagating light of solid state lasers (SSL). While having the advantage that collimation is integrated in on singular optical element, the alignment of this component is very sensitive. With focusing lengths down to $300 \mu \mathrm{m}$ these FAC have to be aligned with an accuracy down to $0.5 \mu \mathrm{m}$ and less. While alignment itself is not as challenging with precise positioning devices available, the stable fixation of the alignment state is more difficult. When polymeric adhesives are used the shrinkage of these adhesives during curing (1\% to $10 \%)$ is one source of dealignment, others are aging due to the continuous radiation load and large thermal ex- 
pansion in the often high power environment of the laser. Replacing the polymeric adhesive by a solder not only increases stability, but also provides better heat transfer away from the component.

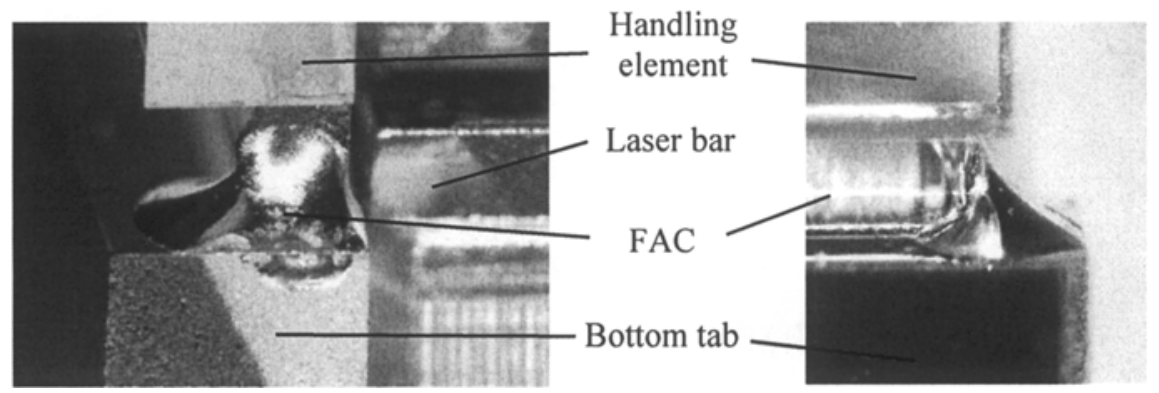

Fig. 6. FAC in front of a laser bar (left: side view, right: front view)

Fig. 6 illustrates how such a FAC can be assembled in front of a laserdiode bar. The FAC in this case is a $11 \mathrm{~mm}$ long aspherical cylinder lens with a diameter of approx. $0.5 \mathrm{~mm}$ and a focal length of $300 \mu \mathrm{m}$. With a working distance of $90 \mu \mathrm{m}$ it is very sensitive in terms of axial and lateral dealignment. This allows for a very precise detection of the FAC behaviour during solder bumping, since in the far field. There micron lateral movements of the FAC are translated into millimetre movements of the collimated beam projected onto a screen, where the beam profile can easily be viewed by a camera (fig. 7). Axial movement (defocus) results in sharpening and desharpening of the beam profile.

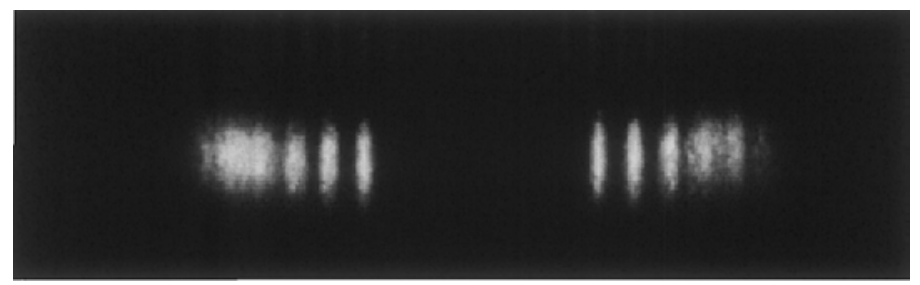

Fig. 7. Camera view of the collimated beam in the far field

To ensure a minimised joining gap between FAC and heat sink of the laser while maintaining 6DOF alignment capability, an additional mounting element ("bottom tab") was introduced. The FAC is joined at its end faces to the bottom tab, while the bottom tab has a planar contact face with respect to the heat sink. This overall leads to a complex sequential joining procedure in which the initially realised alignment state of the FAC gets degraded. This is shown in Fig. 7. The process steps include pre-processing (handling and alignment, steps 1 to 3 ) and post processing (releasing handling equipment, steps 12 to 15). Thus between steps 3 and 12 the solder bumping process chain itself takes place and results in a dealignment of approx. $-0.6 \mu \mathrm{m}$. 


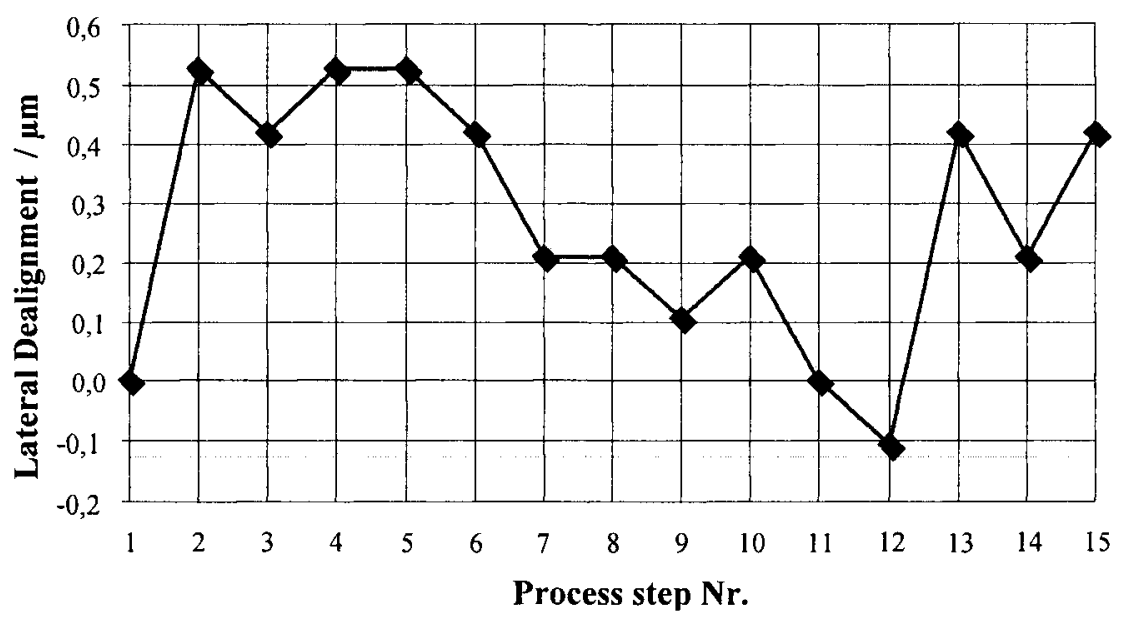

Fig. 8. Dealignment of a FAC during a complex bumping sequence

In a series of six complete FAC processing the dealignment was measured to reproducible in a range of $\pm 0.2 \mu \mathrm{m}$. This allows for pre-compensation of the expected dealignment and further increases joining accuracy

\section{Outlook}

While first experiments proved that solder bumping is a sufficient method to increase repeatability and accuracy compared to conventional flux-free laser beam soldering methods [5], the technology still has to mature for complex precision assembly tasks. Placing accuracy needs to be enhanced in order to use larger bumping distance to reach into complex assembly environments. Long term stability is an issue, since intermetallic diffusion and phase changes are different to what is known from reflow soldering in electronics due to the short laser beam reflow. Most important is a better understanding of the wetting and resolidification process in order to optimise and minimise dealignment during solder bumping, which is still at the limit for some micro-optical applications.

Nevertheless solder bumping technology has a substantial potential for fast varying, high precise assembly processes in microoptics and microsystem technology, where multi-functional and high accuracy packaging techniques are required.

\section{Acknowledgement}

The work presented in this paper was partially funded by the german Federal Ministry of Education and Research within the "Optische Technologien" framework programme (Funding call BrioLas, subproject BriMo, FKZ 13N8612). 


\section{References}

[1] Patent US020040069758A1, "Method and device for applying a solder to a substrate".

[2] E. Zakel, Th. Oppert, G. Azdasht: Laser assisted Wafer Level Packaging for MEMS. In: Proceedings of the Semicon Europa (Munich, Germany), April 19, 2004

[3] S. H. Park, "Robust design and analysis for quality engineering", Chapman\& Hall, London, 1996.

[4] K. J. Puttlitz (editor), "Handbook of lead-free solder technology for microelectronic assemblies", Dekker, New York, 2004.

[5] H. Banse, R. Eberhardt, E. Beckert and W. Stöckl, "Laser Beam Soldering - a New Assembly Technology for Microoptical Systems", Microsystems Technologies 11, 2005, pp.186-193. 